\title{
Terrain reconstruction based on descent images for the Chang'e III landing area
}

\author{
Xu Xinchao ${ }^{1,2 *}$, Zhen Ying ${ }^{1}$, Wang Guobin ${ }^{1}$, Zhou Peixi $^{1}$, Li Hang $^{1}$ and Lee Mengying ${ }^{3}$ \\ ${ }^{1}$ Liaoning Technical University, School of Gematics, Fuxin 123000 China \\ ${ }^{2}$ Institute of Remote Sensing and Digital Earth, Chinese Academy of Sciences, Beijing 100101, China \\ ${ }^{3}$ Information Technology Section of ASSIC Digital Remote Engineering Company, Ottawa, Canada
}

Received 8 February 2015; Accepted 18 October 2015

\begin{abstract}
A new method that combined image matching and shape from shading for terrain reconstruction was proposed to solve the lack of terrain in the landing area of Chang'e III. First, the reflection equation was established based on the LommelSeeliger reflection model. After edge extraction, the gradients of points on the edge were solved. The normal vectors of adjacent points were obtained using the smoothness constraint. Furthermore, the gradients of residual points in the image were determined through evolution. The inadequacy of the reflection equation was eliminated by considering the gradient as the constraint of the reflection equation. The normal vector of each point could be obtained by solving the reflection equation. The terrain without coordinate information was reconstructed by iterating the vector field. After using scaleinvariant feature transform to extract matching points in the descent images, the terrain was converted to a lander centroid coordinate system. Experiments were carried out with MATLAB-simulated images, laboratory images, and descent images of Chang'e III. Results show that the proposed method performs better than the classical SFS algorithm. The new method can provide reference for other deep space exploration activities.
\end{abstract}

Keywords: Descent images, Gradient proportion, Image matching, Landing area, Shape from shading

\section{Introduction}

Efficient path planning is crucial for energy conservation of the Chang'e III rover. High-precision terrain of the landing area is an important basis for efficient path planning [1]. The low-resolution images captured by Chang'e I and Chang'e II in the early years of China's lunar exploration cannot meet the requirements of the path planning task. During landing, descent images were captured by the landing camera in the Chang'e III lander [2-3]. Compared with the previously acquired images, descent images have higher resolution and their orientation can be obtained by inertial navigation [4]. Thus, the conditions for high-precision terrain reconstruction of the landing area are met.

Binocular stereo matching is an established reconstruction method. However, the descent images were acquired with a fixed time interval in the vertical drop process of the lander. Thus, the baseline between descent images was vertical and the image resolutions vary. Reconstructing a 3D terrain by stereo matching technology is difficult and other methods are needed. Shape from shading (SFS) is a 3D reconstruction method in computer vision, but the result is without coordinate information [5].

Brunoa tested an optical technique frequently used in air acquisition, which is based on the projection of structured lighting patterns acquired by a stereo vision system [6]. Xavier completed the reconstruction of non-rigid 3D shapes from stereo-motion [7]. O'Hara developed a new SFS algorithm using a high-resolution stereoscopic camera [8].

* E-mail address: xu_xinchao@163.com ISSN: 1791-2377 @ 2015 Kavala Institute of Technology. All rights reserved.
Kumar presented an approach to reconstruct 3D surfaces by integrating SFS and stereo [9]. Robert completed the reconstruction of $3 \mathrm{D}$ surfaces through image sequences by SFS [10]. Sieu investigated the influence of SFS on the perception of coherent local and global motion using global dot motion stimuli [11]. Grumpe combined photometric image information with high lateral resolution as well as digital elevation model (DEM) data with comparably low lateral resolution and thus obtained lunar DEM with high lateral resolution [12]. Meng reconstructed the surface shape of a landing terrain from descent images by employing scale-invariant feature transform (SIFT), although the accuracy of the results is lower [13]. Dejan studied the effect of contour on SFS [14]. Correal proposed a new system for $3 \mathrm{D}$ terrain reconstruction based on histogram matching [15]. Dessì improved the lighting constraints for SFS [16]. Otero proposed a local iterative modification to address real lens aberrations in 3D reconstruction [17].

Although reconstruction results were obtained by stereo vision, stereo images with horizontal baseline are still required. The reconstruction results of SFS can only be acquired relatively, and these results cannot be used directly in path planning because they have no coordinate information. Path planning requires terrain with real coordinates. Points with real coordinates can be obtained through the feature matching of descent images. Thus, combined with the point coordinates in a real coordinate system and a pixel coordinate system, the terrain of the landing area can be reconstructed using descent images by integrating photogrammetry and SFS technologies. 


\section{SFS Theory}

SFS algorithm is achieved by solving the reflection equation [18-21]. First, the reflection equation of the camera direction, light direction, and surface normal vector of the object is established based on a specific reflection model. After incorporating the directions of the camera and the light, the normal vector of each point on the object surface can be obtained by solving the equation. Finally, the whole shape can be determined by iteration.

The Lommel-Seeliger reflection model is considered to be the closest to the actual reflection situation of the moon [22]. Figure 1 shows a schematic diagram of the LommelSeeliger reflection model, where $i$ is the angle between the camera direction and the surface normal vector, $\mathrm{s}$ is the light incidence angle, $\mathrm{L}$ is the light direction, $\mathrm{S}$ is the camera direction, and $\mathrm{N}$ is the normal vector of the object surface.

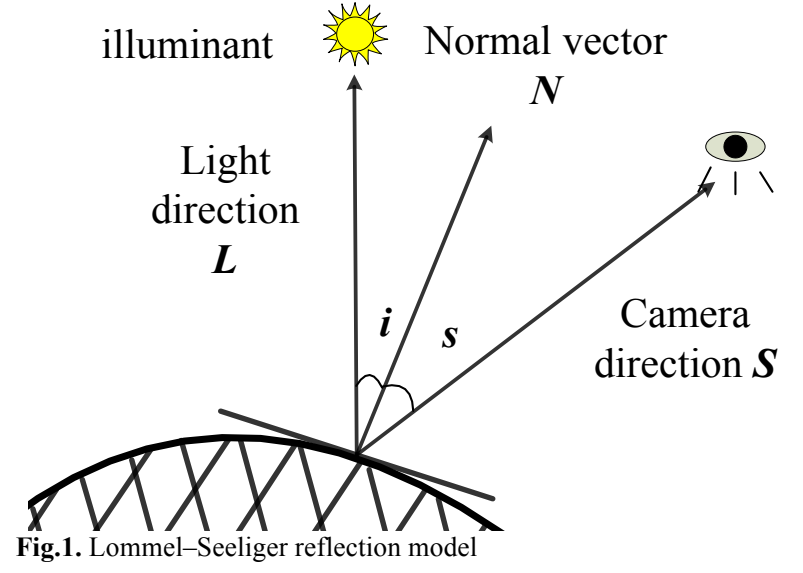

The normal vector of the surface point of the object is assumed to be $N\left(n_{x}, n_{y}, n_{z}\right)$. To reduce the number of unknowns in the reflection equation, the normal vector is converted to gradient form.

$\left\{\begin{array}{l}p=-n_{x} / n_{z}=\partial f(x, y) / \partial x \\ q=-n_{y} / n_{z}=\partial f(x, y) / \partial y\end{array}\right.$

According to Equation (1), the normal vector of the point can be changed to $N(p, q,-1)$, the light vector can be rewritten as $N\left(p_{i}, q_{i},-1\right)$, and the vector of the camera can be transformed to $N\left(p_{s}, q_{s},-1\right)$. The reflection equation can be established based on the Lommel-Seeliger reflection model [8].

$$
I(x, y)=\frac{\omega}{4} \cdot \frac{\cos i}{\cos i+\cos s}
$$

where $(x, y)$ is the row and column of pixels, $I(x, y)$ is the gray value of the corresponding pixel, $\omega$ is the reflectivity of the surface, $\cos i$ is the cosine of the angle between the light direction and the normal vector, and $\cos s$ is the cosine of the angle between the camera direction and the normal vector.

$$
\begin{aligned}
& \cos i=\frac{\left(1+p p_{i}+q q_{i}\right)}{\sqrt{p_{i}^{2}+q_{i}^{2}+1} \cdot \sqrt{p^{2}+q^{2}+1}} \\
& \cos \theta_{s}=\frac{\left(1+p p_{s}+q q_{s}\right)}{\sqrt{p_{s}^{2}+q_{s}^{2}+1} \cdot \sqrt{p^{2}+q^{2}+1}}
\end{aligned}
$$

The normal vector of the point can be determined by solving Equation (2). The normal vector of all image points can be obtained by traversing the entire image. Finally, reconstruction can be completed by iteration based on the obtained vector field. For $3 \mathrm{D}$ reconstruction based on a single image, the results of Pentland and Tsai are better [9].

\section{Description of the Proposed Algorithm}

Clearly, the reflection equation is ill-posed. Thus, additional constraints are needed to complete the normal vector solution. The results obtained by the SFS algorithm are also relative and cannot be used directly for lunar exploration. To solve these problems, a new method of 3D reconstruction based on image matching and SFS is proposed.

\subsection{SFS Regularization}

To complete the $3 \mathrm{D}$ reconstruction, the reflection equation must be regularized first. Edge information, which is an important feature of an image, is crucial in image analysis. Under certain illumination conditions, a change in terrain can alter the image gray, significant changes of gray form of edges in the image. Thus, to some extent, edges can also reflect the changes in terrain, which can be used as a constraint for the SFS algorithm.

To make the problem description convenient, the whole terrain is regarded as a combination of many small blocks that are perpendicular to the edge. The surface of the small blocks can be considered a plane. As shown in Figure 2, the $O-X Y Z$ space rectangular coordinate system is established by considering that the center of the edge of the small blocks is the origin of coordinates, the direction of the $Y$ axis is coincident with the direction of the edge, and the $Z$ axis toward the zenith direction, as well as the $X, Y$, and $Z$ axes satisfy the right-hand rule.

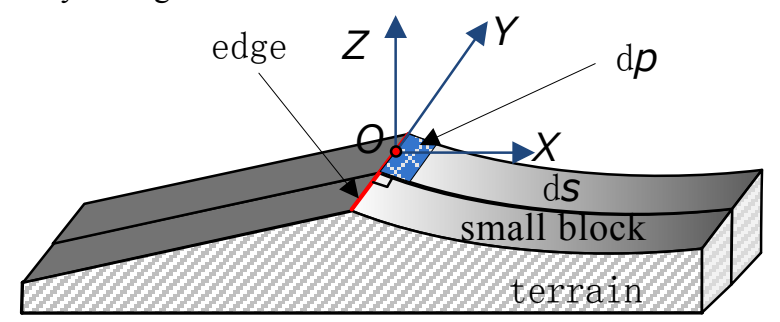

Fig.2. $O-X Y Z$ coordinate system

In the small block, the edge is shorter and can be regarded as a straight line. The projection direction of normal vector in the XY plane is defined as the gradient proportion factor $k$. Assuming that the normal vector of the point $(x, y, z)$ on the edge is $\boldsymbol{N}(p, q,-1), k_{1}$ can be obtained as follows:

$$
k_{1}=q / p
$$

The projection of $\boldsymbol{N}(p, q,-1)$ on the $\mathrm{XY}$ plane is clearly $N(p, 0)$. Thus, gradient proportion factor $k_{1}$ of the point $(x, y, z)$ is equal to 0 .

The small plane $\mathrm{d} p$ is adjacent to the edge. The angle between $\mathrm{d} p$ and the XY plane is $\theta$. Evidently, $0<\theta<90$. The normal equation of plane $\mathrm{d} p$ under the $O-X Y Z$ coordinate system can be described as

$-\sin \theta x+\cos \theta z=0$ 
By solving the Equation (6), the normal vector of any point in plane $\mathrm{d} p$ can be obtained. The normal vector of the points adjacent to the edge can be described as $N(\sin \theta / \cos \theta, 0,-1)$, and the gradients proportion factor $k_{2}$ is

$k_{2}=q / p=0 /(-\sin \theta / \cos \theta)=0$

Thus, the projection of points on the edge and on plane $\mathrm{d} p$ is the same as in the XY plane $\left(k_{1}=k_{2}=0\right)$. Other small blocks can also be tested by employing the method mentioned above. Some points are assumed to exist in plane $\mathrm{d} p$ that are adjacent to the edge. Thus, the gradient proportion of those points can be obtained through the gradient of the adjacent edge points, which can be called the gradient proportion constraint.

After the extraction of the edge in the entire image, the gradient proportion factor of the points on the edges can be solved after fitting. The gradient proportion factors of all the points adjacent to the edges can be obtained according to the gradient proportion constraint.

In general, terrain surfaces are considered continuous. Assuming that the coverage of the image $(x, y)$ is $\Omega$, the continuous terrain surface model can be expressed as follows:

$$
\Delta=\iint_{\Omega}\left(p_{x}^{2}+p_{y}^{2}+q_{x}^{2}+q_{y}^{2}\right) \mathrm{d} x \mathrm{~d} y
$$

where $p_{x}$ and $p_{y}$ are the partial derivatives of $p$ in the $\mathrm{X}$ and $\mathrm{Y}$ directions, and $q_{x}$ and $q_{y}$ are the partial derivatives of $q$ in the $\mathrm{X}$ and $\mathrm{Y}$ directions respectively.

Equation 8 is called the continuity constraint. Under this condition, the normal vector of each point on the object surface is close. Thus, to complete the quantitative constraints of the reflection equations for all the points in the whole image, the gradient proportion factors of the edge adjacent points can be solved first. Next, the gradient proportion factors of the remaining points in the image can be evolved under the continuity constraint. Finally, the quantitative constraints for the entire image can be completed.

\subsection{Coordinate Solution of Control Points}

After obtaining the gradient proportion constraint, the illposed nature of the reflection equation is eliminated. However, the SFS solution can only be determined relatively and has no practical coordinate meaning. Path planning of "Jade Rabbit" lunar rover requires the terrain to be under the coordinate system, which considers the centroid of the lander as the origin of the coordinate system. Thus, the SFS results can be used directly in path planning after being constrained by other methods. Although these results cannot be used to complete the reconstruction through the traditional stereo vision method, highly accurate and reliable matching points can be obtained by employing feature matching, which can provide the coordinate constraints for the SFS reconstruction results.

The descent images are obtained by the landing camera at the bottom of the lander in the vertical descent. The exterior orientation elements of the images include position factor $\left(X_{s 1}, Y_{s 1}, Z_{s 1}\right)$, tilt angle of image $(\varphi)$, slant angle of image $(\omega)$, and rotation angle of image $(\kappa)$, which can be directly obtained using inertial measurement equipment and a laser altimeter sensor. The focal length of the landing camera is $\mathrm{f}$, and the pixel coordinates of the point are $(x, y)$. The ground coordinates of the matching points to be solved are $(X, Y, Z)$. The collinearity equation can be established according to the above parameters.

$$
\begin{aligned}
& (x)=-f \frac{a_{1}\left(X-X_{s}\right)+b_{1}\left(Y-Y_{s}\right)+c_{1}\left(Z-Z_{s}\right)}{a_{3}\left(X-X_{s}\right)+b_{3}\left(Y-Y_{s}\right)+c_{3}\left(Z-Z_{s}\right)} \\
& (y)=-f \frac{a_{2}\left(X-X_{s}\right)+b_{2}\left(Y-Y_{s}\right)+c_{2}\left(Z-Z_{s}\right)}{a_{3}\left(X-X_{s}\right)+b_{3}\left(Y-Y_{s}\right)+c_{3}\left(Z-Z_{s}\right)}
\end{aligned}
$$

The descent image is captured vertically. Thus, the attitude parameters $\varphi=\omega=0$. Assuming that the exterior orientation elements of image 1 are $\left(X_{s 1}, Y_{s 1}, Z_{s 1}, 0,0, \kappa_{1}\right)$, the exterior orientation elements of image 2 are $\left(X_{s 2}, Y_{s 2}, Z_{s 2}, 0,0, \kappa_{2}\right)$. The pixel coordinates of the matching point acquired from images 1 and 2 by SIFT are $(\mathrm{x} 1, \mathrm{y} 1)$ and $(\mathrm{x} 2, \mathrm{y} 2)$ [23]. Incorporating the coordinates of the matching points and exterior orientation elements of images 1 and 2 into Equation (9) establishes the following equations:

$$
\left.\begin{array}{l}
l_{11} X+l_{12} Y+l_{13} Z-l_{x 1}=0 \\
l_{14} X+l_{15} Y+l_{16} Z-l_{y 1}=0 \\
l_{21} X+l_{22} Y+l_{23} Z-l_{x 2}=0 \\
l_{24} X+l_{25} Y+l_{26} Z-l_{y 2}=0
\end{array}\right\}
$$

where

$$
\begin{aligned}
& l_{11}=f \cos \kappa_{1}, l_{12}=f \sin \kappa_{1}, l_{13}=x_{1} \\
& l_{14}=-\sin \kappa_{1} f, l_{15}=f \cos \kappa_{1}, l_{16}=y_{1} \\
& l_{x 1}=f \cos \kappa_{1} X_{s 1}+f \sin \kappa_{1} Y_{s 1}+x_{1} Z_{s 1} \\
& l_{y 1}=-\sin \kappa_{1} f X_{s 1}+f \cos \kappa_{1} Y_{s 1}+y_{1} Z_{s 1} \\
& l_{21}=f \cos \kappa_{2}, l_{22}=f \sin \kappa_{2}, l_{23}=x_{2} \\
& l_{24}=-\sin \kappa_{2} f, l_{25}=f \cos \kappa_{2}, l_{26}=y_{2} \\
& l_{x 2}=f \cos \kappa_{2} X_{s 2}+f \sin \kappa_{2} Y_{s 2}+x_{2} Z_{s 2} \\
& l_{y 2}=-\sin \kappa_{2} f X_{s 2}+f \cos \kappa_{2} Y_{s 2}+y_{2} Z_{s 2}
\end{aligned}
$$

The 3D coordinates of the matching points under the lander centroid coordinate system can be determined by incorporating Equations (11) and (12) into Equation (10).

\subsection{Control Point Selection}

Solving Equation (10) can obtain the coordinates of the matching points in descent images. The coordinate accuracy of the matching points in different positions of the sequence images varies. Thus, before the constraint of the SFS results is transformed, the matching points must be selected first and the points with higher precision considered as the control points for constraint transformation. The coordinate of points far from the image center has higher precision [24]. Based on the above characteristics, the following two principles are considered in the final selection of control points:

(1) Considering the texture characteristics of the lunar surface, the more obvious feature point in the image should be selected as the control point.

(2) The point far from the image center should be selected. 


\subsection{Algorithm Implementation}

First, the SFS method is carried out for the reconstruction and the shape is obtained through iteration. The matching points of descent images are identified through SIFT, and the coordinates under the lander centroid coordinate system can be acquired by combining the exterior orientation elements of the image. The matching points are used as the control points after being selected. Finally, the SFS results are converted to the terrain, which can be directly used in the path planning task. The details of this process are as follows:

(1) Two images are selected in the descent images, and the corresponding exterior orientation elements are obtained simultaneously.

(2) The edge, which has a smaller coverage, is extracted through Canny algorithm in the image [25]. The edges are fitted, and the gradient proportion of each point on the edges is calculated.

(3) First, the gradient proportions of the points adjacent to the edges are obtained based on the gradient proportion constraint. Next, the gradient proportions of the rest of the points in the image can be solved.

(4) The reflection equation is established for each pixel based on the Lommel-Seeliger reflection model. After obtaining the regularization constraint of the gradient proportion, the normal vector of the point corresponding to the pixel can be solved using the reflection equation.

(5) According to the normal vector of all the points, iteration is used to accomplish the reconstruction by SFS.

(6) The matching points can be obtained after extracting the feature points in two images using the SIFT method. The points are selected according to the selection principle of the matching points. When the matching results are considered as the control points and the matching results and exterior orientation elements of the image are combined, the coordinates of the selected matching points under the lander centroid coordinate system can be obtained.

(7) Combining the coordinates of control points in the SFS results and the lander centroid coordinate system can solve seven parameters of the Bursa model. Finally, the reconstruction is completed by employing the seven parameters to convert the SFS results. The terrain can be directly used in lunar exploration.

\section{Experimental Results and Discussion}

To assess the accuracy of the proposed algorithm for reconstruction, three types of data, namely, MATLAB simulated images, laboratory images, and images of Chang'e III, are used.

\subsection{MATLAB-Simulated Images}

MATLAB-simulated images are used to verify the correctness of the SFS portion of the proposed algorithm. The illumination direction of images 1 and 2 are $(1.5,0,1)$ and $(1,0.8,1.5)$ respectively.

Figure 3a shows image 1 simulated by MATLAB, Figure $3 \mathrm{~b}$ presents the real shape that corresponds to image 1, Figure $3 \mathrm{c}$ describes image 2 simulated by MATLAB, and Figure $3 \mathrm{~d}$ illustrates the real shape that corresponds to image 2.

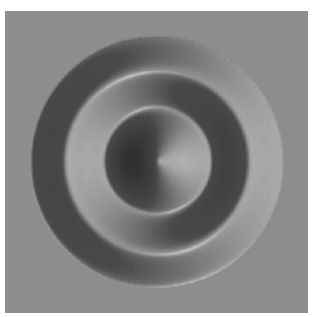

(a)

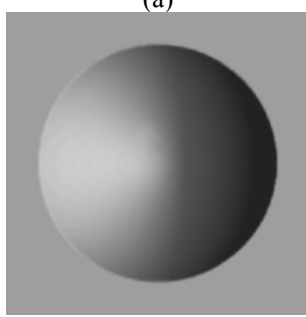

(c)

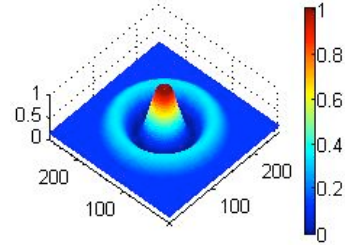

(b)

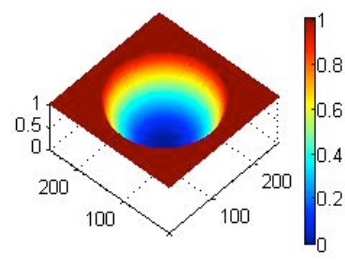

(d)
Fig.3. MATLAB-simulated images and their shape

The Pentland and Tsai methods, which exhibit better recovery effect in SFS, and the proposed methods are employed to restore two images. Figures 4 and 5 are the reconstruction results of images 1 and 2 respectively. (a), (b), and (c) are the reconstruction results of the Pentland algorithm, Tsai algorithm, and proposed algorithm, respectively.

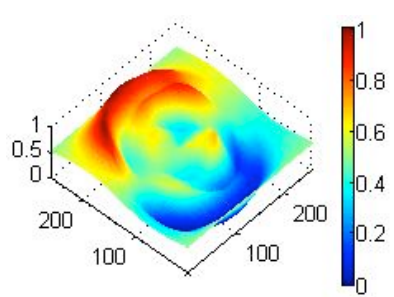

(a)

Fig.4. Reconstruction results of image 1

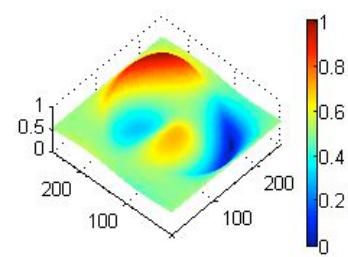

(a)

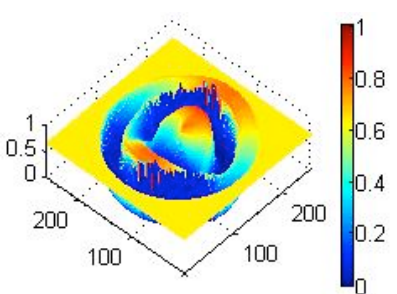

(b)

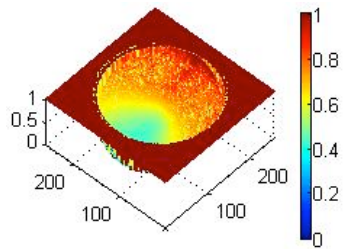

(b)

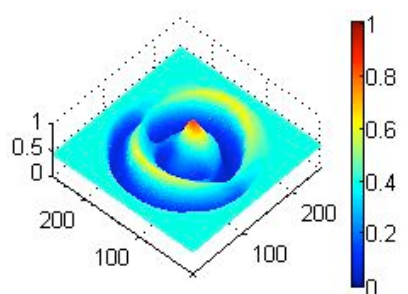

(c)

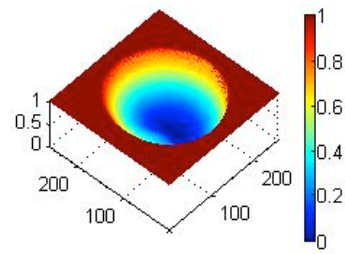

(c)

Fig.5. Reconstruction results of image 2 
The reconstruction error of various methods is obtained based on the actual shape of the simulated image. Figures 6 and 7 show the reconstruction errors of images 1 and 2 under different methods. (a), (b), and (c) are the reconstruction errors of the Pentland algorithm, Tsai algorithm, and proposed algorithm, respectively.

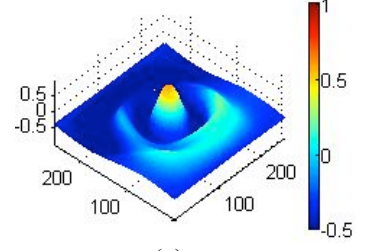

(a)

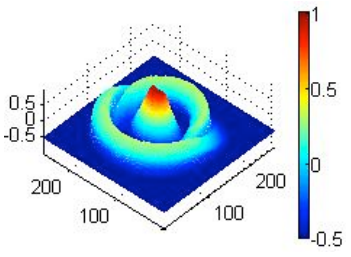

(b)

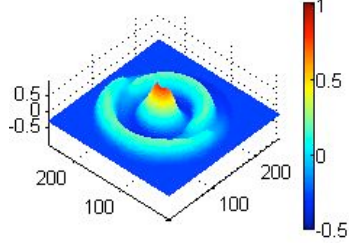

(c)

Fig.6. Reconstruction errors of image 1 under different methods

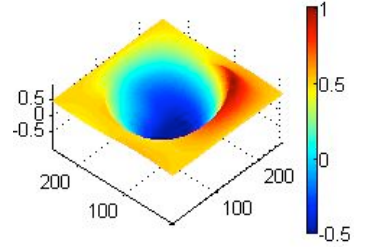

(a)

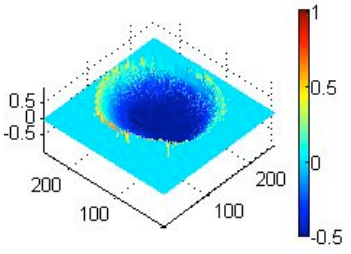

(b)

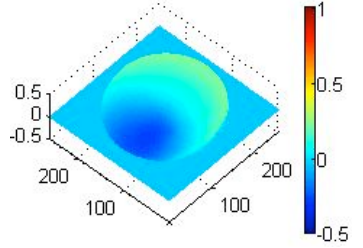

(c)

Fig.7. Reconstruction errors of image 2 under different methods

The charts of the reconstruction results and the reconstruction errors can effectively verify the feasibility of our algorithm. Tables 1 and 2 indicate the statistical values of the reconstruction errors. The SFS reconstruction results are relative. Thus, the errors are reflected in a uniform scale without a specific unit.

Table 1. Error statistics of image 1

\begin{tabular}{ccccc}
\hline Algorithm & Minimum & Maximum & Mean & Mean square \\
\hline Pentland method & -0.851 & 0.871 & 0.341 & 0.200 \\
Tsai method & -0.867 & 0.955 & 0.380 & 0.180 \\
Proposed method & -0.370 & 0.777 & 0.217 & 0.102 \\
\hline
\end{tabular}

Table 2. Error statistics of image 2

\begin{tabular}{ccccc}
\hline Algorithm & Minimum & Maximum & Mean & Mean square \\
\hline Pentland method & -0.627 & 0.893 & 0.291 & 0.203 \\
Tsai method & -0.782 & 0.802 & 0.151 & 0.207 \\
Proposed method & -0.266 & 0.286 & 0.080 & 0.070 \\
\hline
\end{tabular}

The results can be obtained by employing statistical tools. In all the SFS reconstruction results, large errors tend to occur in the shadow and in poorly illuminated areas. SFS is a reconstruction algorithm based on pixel gray. Thus, the reflection of the shadow and the poorly illuminated area is not consistent with the reflective nature of the theory. The accuracy of the SFS recovery results in this type of area is poor and cannot even be recovered. Reconstructing this type of area cannot be completed with binocular stereo vision methods.

The Pentland method recovery error occurs mainly in areas where gray changes in the image are more intense. This phenomenon occurs because $p$ and $q$ in the reflection equation are carried out by Fourier transformation and linearization by Pentland, and the result is inhibited by the smoothness constraint.

The results of the Tsai method are better in wellilluminated areas. However, in the shadow region, particularly near the edge of the shadow, the results of this method significantly vary from actual conditions. The main reason for this observation is that the reflection equation is linearized by the Tsai algorithm, and the reflection of the edge is changed from the ideal reflection model. Thus, substantial error in the final reconstruction results appears.
The results of the proposed algorithm are closer to the actual situation because the gradient directions of the edge are used to complete the constraints for the normal vector of the terrain points. Thus, our method is better than the previous methods. The maximum error and the mean square error are smaller, although the error of the shadow area recovery is still large.

\subsection{Laboratory Images}

Laboratory images are used to verify the accuracy of the overall algorithm. Figure 7 shows the laboratory images of the simulated landing process, which are captured at different heights. The light directions are (1, 0, 0.7). (a), (b), (c), and (d) are images 3 to 6 , respectively. Image 3 is taken at the height of $11 \mathrm{~m}$, image 4 at $8 \mathrm{~m}$, image 5 at $5.5 \mathrm{~m}$, and image 6 at $4 \mathrm{~m}$. Riegl 360i 3D laser scanner is employed to scan the simulated lunar surface in the laboratory, and the scan interval is $0.5 \mathrm{~cm}$. The accuracy of the results of different algorithms is tested by considering the scan results as correct.

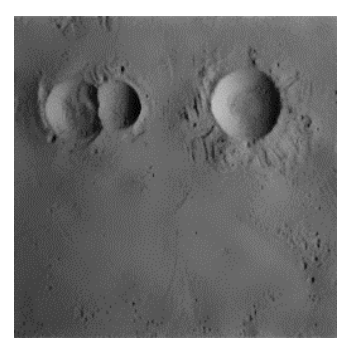

(a)

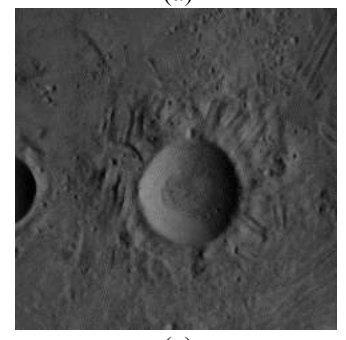

(c)

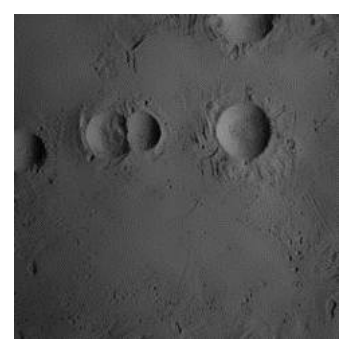

(b)

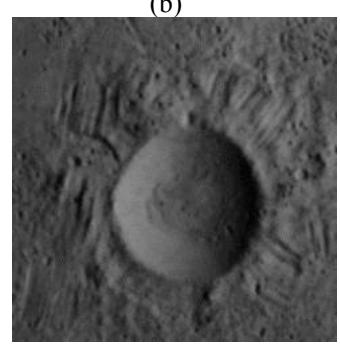

(d)
Fig. 7. Laboratory images

Adopting the proposed selection principle can obtain the matching points through the SIFT algorithm, as shown in 
Figure 8. (a) is the matching result of images 3 and 4 , and (b) is the matching result of images 5 and 6 .
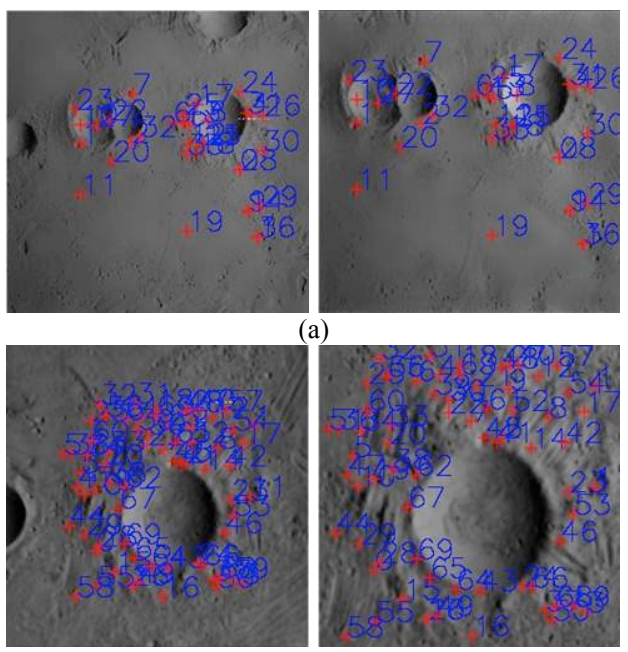

(b)

Fig. 8. Matching results

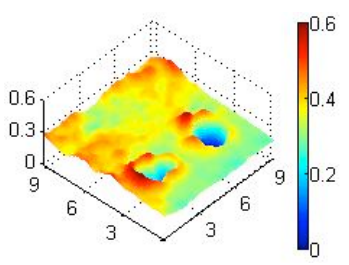

(a)

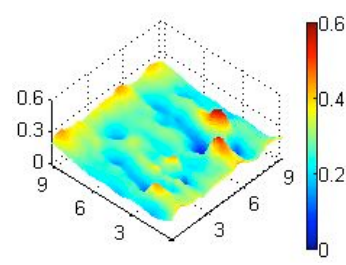

(b)

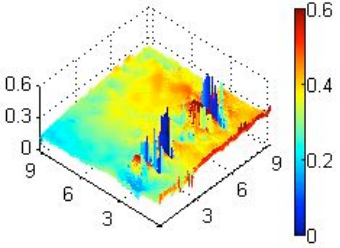

(c)

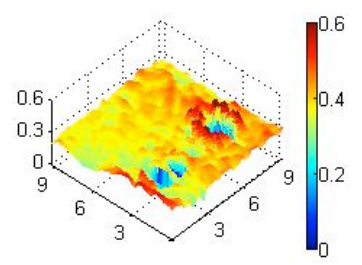

(d)

Fig.9. Reconstruction results of image 4

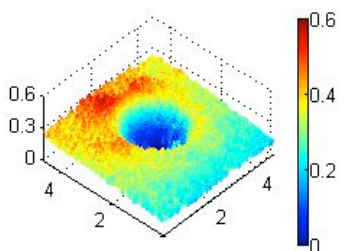

(a)

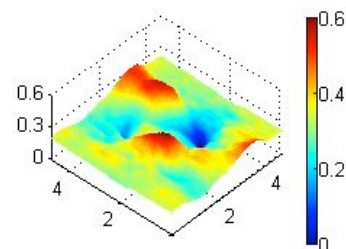

(b)

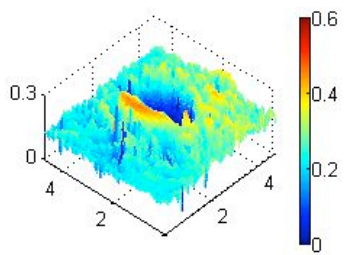

(c)

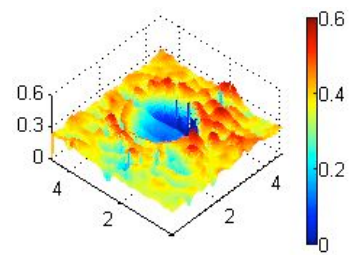

(d)

Fig.10. Reconstruction results of image 6

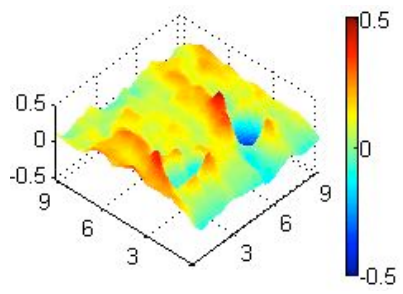

(a)

Fig.11. Reconstruction errors of image 4

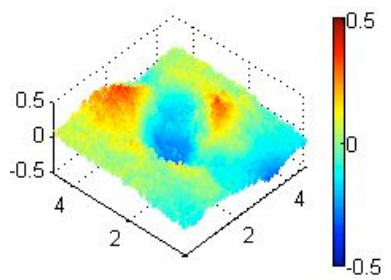

(a)

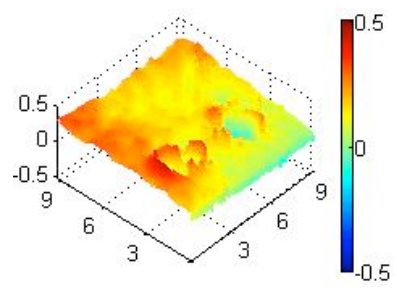

(b)

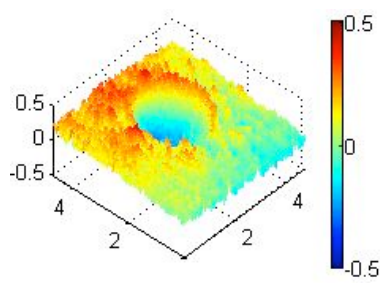

(b)

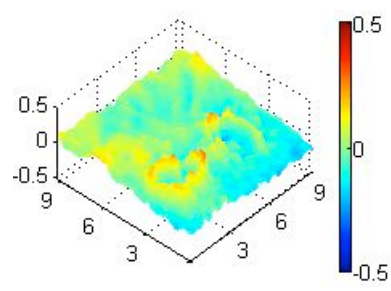

(c)

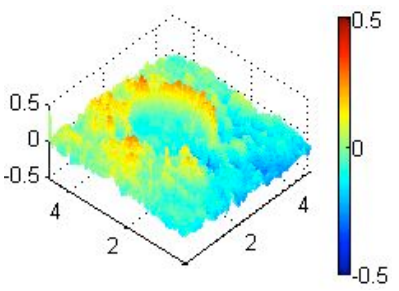

(c)

Fig.12. Reconstruction errors of image 6 
Tables 3 and 4 present the statistical values of the reconstruction errors of images 4 and 6 under different methods respectively.

Table 3. Error statistics of image 4

\begin{tabular}{cllll}
\hline Algorithm & Minimum & Maximum & Mean & Mean square \\
\hline Pentland method & -0.481 & 0.485 & 0.111 & 0.076 \\
Tsai method & -0.418 & 0.452 & 0.157 & 0.074 \\
Proposed method & -0.431 & 0.451 & 0.067 & 0.050 \\
\hline
\end{tabular}

Table 4. Error statistics of image 6

\begin{tabular}{cllll}
\hline Algorithm & Minimum & Maximum & Mean & Mean square \\
\hline Pentland method & -0.361 & 0.382 & 0.100 & 0.078 \\
Tsai method & -0.340 & 0.467 & 0.120 & 0.085 \\
Proposed method & -0.315 & 0.471 & 0.094 & 0.063 \\
\hline
\end{tabular}

Based on the reconstruction results and the errors of different algorithms in the figures and tables above, the following results can be obtained:

(1) Compared with the classical SFS algorithm, the recovery results of the proposed method exhibit the correct size after obtaining the constraint of the matching point (unit: $\mathrm{m})$. Such reconstruction result can be used for the path planning of the rover.

(2) The proposed algorithm exhibits the smallest maximum error compared with the Pentland and Tsai algorithms, and the mean error and mean square error are lesser than that of the previous algorithm. Thus, the proposed algorithm is better in terms of reducing errors.

(3) In the reconstruction results for laboratory images, the maximum errors of the three methods occur in the shadow areas. In addition, the errors near the edges are greater than

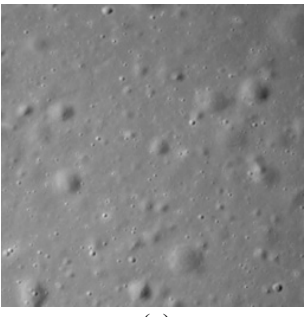

(a)

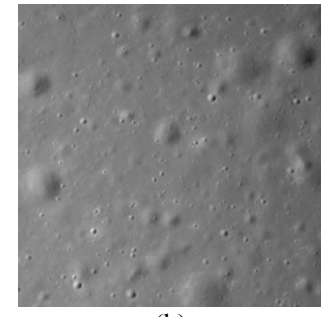

(b)

those in other areas. These phenomena occur because the reflection characteristics of these areas vary from the theoretical reflection model.

(4) Global errors exist because of the errors of illumination, parameters for coordinate system transformation, and reflection coefficient of the material.

(5) Surface continuity constraint is used in the Pentland method and the proposed method. The results of the two algorithms are smoother compared to the recovery results of the Tsai algorithm.

(6) In the proposed algorithm, a large error occurs in the shadow and in areas near the edges, indicating that the positioning accuracy of the edges significantly affects the final results. The errors of gradient proportion are also attributed to the edge position. Edge position caused the difference between the final results and the actual terrain.

\subsection{Images of Chang'e III}

Two images of Chang'e III were extracted for the reconstruction. Figures $13 \mathrm{a}$ and $13 \mathrm{~b}$ illustrate the descent images of the Chang'e III lander, and the matching points obtained by the SIFT algorithm are shown in Figure 13c. According to the shooting time of the image, the light direction can be obtained, which is $(1,-0.5$, and 0.8$)$. The shooting height of image 7 was $33 \mathrm{~m}$, and the shoot height of image 8 was $20 \mathrm{~m}$.

Reconstructions are first carried out using the three SFS methods, and the results are converted by the matching points. Figure 14 presents the reconstruction results of different methods. (a) is the Pentland result, (b) is the Tsai result, and (c) is the result of the proposed method.
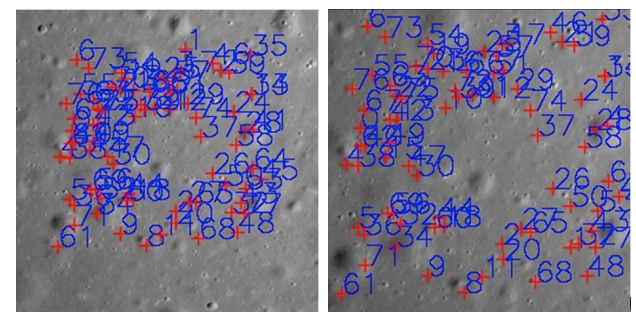

(c)

Fig. 13. Descent images and matching result

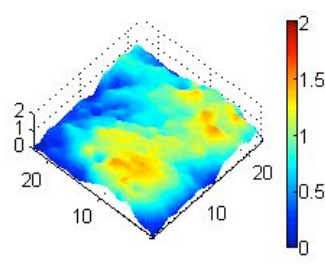

(a)

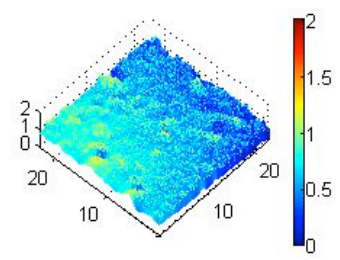

(b)

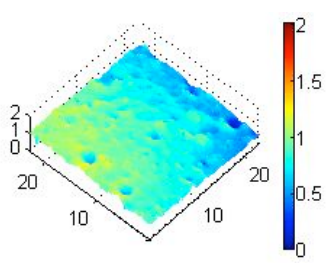

(c)
Fig. 14. Reconstruction results of Chang'e III image

The results show that the Pentland result is the worst among all three methods, the result of Tsai is better, and the result of the proposed method is closest to the actual terrain.

\subsection{Analysis}

The proposed algorithm uses gradient proportion constraint instead of the traditional mathematical constraint. Thus, our SFS terrain reconstructions are closer to the actual situation. The SFS reconstruction result was converted by the matching points, which can be used directly in practical engineering.

The main factors of this paper are as follows:
(1) The gradient proportional constraint is the primary factor in our algorithm. Gradient proportional factor is solved starting from the edges. The positions of the extracted edges vary from actual positions, resulting in the error of the normal vector and reconstruction error eventually.

(2) After fitting the edge, the gradients of points on the edge can be obtained. The gradients of the rest of the points can be solved through evolution. The number of pixels used determines the accuracy of edge fitting, which finally affects the gradient proportion.

(3) The coordinates of the matching points under the lander centroid coordinate system in descent images can be solved by combining the exterior orientation elements. The 


\section{Xu Xinchao, Zhen Ying, Wang Guobin, Zhou Peixi, Li Hang and Lee Mengying}

/Journal of Engineering Science and Technology Review 8 (4) (2015) 56- 63

corresponding coordinates in the SFS result can be determined. The coordinates of these points are used to solve the transformation parameters for the coordinate system. The errors of these coordinates result in the error of the conversion parameters.

\section{Conclusions}

This paper presents a new reconstruction approach combined with image matching and SFS. The projection direction of the normal vector of the surface point and adjacent edge point is the same in the plane. According to the proposed principle of uniform projection direction, the reflection equation could be constrained by the gradient proportion instead of the traditional mathematical method. Thus, the 3D reconstruction results could be obtained relatively. The results were restricted by the matching points and could be converted to the lander centroid coordinate system. The results of testing the proposed approach demonstrated that the reconstruction errors of the proposed method were lesser than those of the Pentland and Tsai methods. The result of the proposed algorithm for descent images of Chang'e III was closer to the actual situation. The proposed method combines image matching and SFS technology for the first time. This method addresses the weakness of the traditional SFS algorithm, which can only obtain the relative shape, overcomes the deficiency of the matching point caused by the lack of texture in the descent images, and successfully applies the matching of descent images of Chang'e III. The proposed algorithm of this work can serve as a reference for China's lunar exploration project.

\section{Acknowledgements}

This work was supported by National Natural Science Foundation of China (No. 41401535) and College Student Innovation and Entrepreneurship Training Program (No. 201410147016).

\section{References}

1. Wu, W. R., Zhou, J. L., Wang, B.F., and Liu, C. K., "Key technologies in the teleoperation of Chang'E-3" Jade Rab-bit" rover", Science China Informations, 44 (4), 2014, pp.425-440.

2. Jia, Y., Liu, S. C., Li, M.L., Li, Q.Z., Peng, S., Wen, B., Ma,Y. Q., and Zhang, S., "Chang'E-3 system pinpoint landing localization based on descent image sequence", Chinese Science Bulletin (Chin Version), 59(19), 2014, pp.1838-1843.

3. Liu, S.C., Jia, Y., Ma, Y.Q., Gu, Z., Wei, S.Y., Li, Q.Z., Xu, X.C.,Wen, B., Zhang, S., Li, M.L., Ma, H., and Yang, H., "High precision localization of the Chang'E-3 lunar rover", Chinese Science Bulletin (Chinese Version), 60(4), 2015, pp. 372-378.

4. Liu, B. Xu, B. Liu, Z.Q. Liu, Y. L., Di, K. C., Tang, G. S., and Zhou, J. L., "Descending and landing trajectory recovery of Chang'e-3 lander using descent images", Journal of Remote Sensing , 18(5), 2014, pp.981-987.

5. Vogel, O., Valgaerts, L., Breub, M., and Weickert, J., "Making Shape from shading work for real-world Images", Pattern Recognition, 567(3), 2009, pp.733-744.

6. Brunoa, F., Bianco, G., Muzzupappaa, M., Barone, S., and Razionale, A.V., "Experimentation of structured light and stereo vision for underwater 3D reconstruction", Journal of Photogrammetry and Remote Sensing, 66(4),2011,pp. 508-518.

7. Lladó, X., Bue, A. D., Oliver, Ar., Salvi, J., and Agapito, L., "Reconstruction of non-rigid 3D shapes from stereo-motion", Pattern Recognition Letters, 32(7), 2011, pp. 1020-1028.

8. O'Hara, R., and Barnes, D., "A new shape from shading technique with application to Mars Express HRSC images", Journal of Photogrammetry and Remote Sensing, 67(1), 2012, pp.27-34.

9. Kumar, S., and Kumar, M., "Application of neural network in integration of shape from shading and stereo", Journal of King Saud University-Computer and Information Sciences, 24(2), 2012, pp.129-136.

10. Cook, R. G., Muhammad, A. J., Kierse, A., Nicholas, C., and Commons-Miller, N., "Shape from shading in pigeons", Cognition, 124(3), 2012, pp.284-303.

11. Khuu, S. K., and Khambiye, S., "The influence of shape-fromshading information on the perception of global motion", Vision Research, 55(4), 2012, pp.1-10.

12. Grumpe, A., Belkhir, F., and Wo"hler, C., "Construction of lunar DEMs based on reflectance modelling", Advances in Space Research, 53(12), 2014, pp.1735-1767.
13. Meng, C., Zhou, N., Xue, X. L., and Jia, Y., "Homography-based depth recovery with descent images", Machine Vision and Applications, 24(5), 2013, pp.1093-1106.

14. Todorovic', D., "How shape from contours affects shape from shading", Vision Research, 103(10), 2014, pp.1-10.

15. Correal, R., Pajares, G., and Ruz, J. J., "Automatic expert system for 3D terrain reconstruction based on stereo vision and histogram matching", Expert Systems with Applications, 41(4), 2014, pp. 2043-2051.

16. Dessì, R., Mannu, C., Rodriguez, G., Tanda, G., and Vanzi, M., "Recent improvements in photometric stereo for rock art 3D imaging", Digital Applications in Archaeology and Cultural Heritage. (7), 2015, pp.1-8.

17. Otero, J., and Sánchez, L., "Local iterative DLT soft-computing vs. interval-valued stereo calibration and triangulation with uncertainty bounding in 3D reconstruction", Neurocomputing, 167(21), 2015, pp.44-51.

18. Khuu, S., and Khambiye, S., "Recovering Shape by Shading and Stereo Under Lambertian Shading Model", International Journal of Computer Vision, 85(1), 2009, pp.58-100.

19. Lopez-Molina, C., Baets, D. B., Barrenechea, E., and Bustince, H., "Edge detection on interval-valued images", Advances in Intelligent and Soft Computing, 107(2), 2012, pp. 325-337.

20. $\mathrm{Xu}, \mathrm{B}$. , and $\mathrm{Li}, \mathrm{H}$., "Surface image edge detection algorithm based on grey relational analysis", Advanced Materials Research, 461(2), 2012, pp.343-346.

21. Yang, L., Ma, S.W., and Tian, B., "New shape-from-shading method with near-scene point lighting source condition", Advances in Intelligent and Soft Computing, 122(2), 2012, pp.653-664.

22. McEwen, A. S., "A precise lunar photometric function", Lunar and Planetary Science, 27(3), 1996, pp.841-842.

23. Lowe, D.G., "Distinctive image features from scale-invariant key points", International Journal of Computer Vision, 60(2), 2004, pp.91-110.

24. Tao, K., Zhang, Z.X., and Zhang, J. Q., "Panning and Multibaseline Digital Close-range Photogrammetry", Geomatics and Information Science of Wuhan University, 34(1), 2009, pp.44-48.

25. Neto, E., Gomesa, S., and Filho, P., "Albuquerqueb V. Brazilian vehicle identification using a new embedded plate recognition system”, Measurement, 70(14), 2015, pp.36-46. 